\title{
REAL ZEROS OF DERIVATIVES OF MEROMORPHIC FUNCTIONS AND SOLUTIONS OF SECOND ORDER DIFFERENTIAL EQUATIONS
}

\author{
BY
}

SIMON HELLERSTEIN, ${ }^{1}$ LI-CHIEN SHEN AND JACK WILLIAMSON

\begin{abstract}
We classify all functions $F$ meromorphic in the plane with only real zeros and real poles which satisfy the additional conditions that $F^{\prime}$ has no zeros and $F^{\prime \prime}$ only real zeros. We apply this classification, in combination with some earlier results, to the study of the reality of zeros of solutions of the equation $w^{\prime \prime}+H(z) w=0, H$ entire.
\end{abstract}

Introduction and statement of the main results. In a series of papers [3, 4,6 the authors recently settled an old conjecture of Pólya by characterizing those entire functions $f$ for which $f, f^{\prime}$ and $f^{\prime \prime}$ have only real zeros. These results may be summarized by

THEOREM A. Suppose that $f$ is entire and $f, f^{\prime}, f^{\prime \prime}$ have only real zeros. Then $f$ is either a Laguerre-Pólya function, i.e., has the form

$$
f(z)=A z^{m} e^{-a z^{2}+b z} \Pi\left(1-z / z_{n}\right) e^{z / z_{n}},
$$

where $A$ is a constant, $a \geq 0, b$ and the $z_{n}$ are real, and $\sum z_{n}^{-2}<\infty$, or $f$ has one of the forms

$$
\begin{aligned}
& f(z)=A e^{B z} \\
& f(z)=A\left(e^{i c z}-e^{i d}\right) \\
& f(z)=A \exp \left(e^{i(c z+d)}\right) \\
& f(z)=A \exp \left\{K\left[i(c z+d)-e^{i(c z+d)}\right]\right\}
\end{aligned}
$$

where $A, B, K, c$ and $d$ are constants, $A c \neq 0, B$ nonreal, $K, c$ and $d$ real, $-\infty<$ $K \leq-1 / 4$.

Observe that the functions given by (0.1) are (constant multiples of) real entire functions, while those of the form $(0.2)-(0.5)$ are strictly nonreal entire functions ( $f$ is real if $z$ real implies $f(z)$ real, while $f$ is strictly nonreal if it is not a constant multiple of a real function).

In [6] we also characterized the strictly nonreal meromorphic functions $F$ having only real poles (and at least one) for which $F, F^{\prime}$ and $F^{\prime \prime}$ have only real zeros. We showed the following theorem.

Received by the editors November 1, 1983.

1980 Mathematics Subject Classification. Primary 34A20, 30D35; Secondary 34C10, 34A30.

${ }^{1}$ Supported in part by NSF grant MCS-800318. 
THEOREM B. Suppose that $F$ is strictly nonreal, meromorphic, not entire, with only real poles. If $F, F^{\prime}$ and $F^{\prime \prime}$ have only real zeros, then

$$
F(z)=A e^{-i(c z+d)} / \sin (c z+d)
$$

or

$$
F(z)=A \exp \left[-2 i(c z+d)-2 e^{2 i(c z+d)}\right] / \sin ^{2}(c z+d),
$$

where $A, c$ and $d$ are constants, $A c \neq 0, c$ and $d$ real.

We have been unable to classify all real meromorphic $F$ with real poles, for which $F, F^{\prime}$ and $F^{\prime \prime}$ have only real zeros. Although this seems to us to be a very hard problem, our attempts have led us to

CONJECTURE 1. Let $F$ be real, transcendental, meromorphic, not entire, with only real simple poles. If $F, F^{\prime}$ and $F^{\prime \prime}$ have only real zeros, then

$$
F(z)=A \tan (a z+b)+B
$$

or

$$
F(z)=A[\tan (a z+b)-C z-D],
$$

where $A, a, b, C$ and $D$ are real constants, $A a C \neq 0, C$ and $D$ suitably restricted.

Indeed, the conjecture is true in the special case where $F$ is the reciprocal of a real entire function having only real zeros and at least one. Results of Hellerstein and Williamson [5] and Rossi [12] give

THEOREM C. Let $f$ be real entire with only real zeros and at least one. If $F=1 / f$ and $F^{\prime}, F^{\prime \prime}$ have only real zeros, then $F(z)=(A z+B)^{-n} ; A$ and $B$ real constants, $A \neq 0, n$ a positive integer.

Before stating our main result, we remark that settling Conjecture 1 would not only provide a desired characterization of one class of meromorphic functions, but it would also shed some interesting light on Pólya's "final set" theorem for this class of functions. This elegant theorem $[\mathbf{1 1}]$ describes in a simple geometrical way the set of accumulation points of all zeros of all of the functions $F, F^{\prime}, F^{\prime \prime}, \ldots$ (i.e. the final set of $F$ ) solely in terms of the location of the poles of $F$. In the case that $F$ has only real poles, and at least two that are distinct, the final set of $F$ consists of the perpendicular bisectors of the segments joining consecutive (distinct) poles of $F$. If, in fact, the only transcendental $F$ having only real simple poles for which $F, F^{\prime}$ and $F^{\prime \prime}$ have only real zeros are those $F$ of the form $(0.6)-(0.9)$ then, since (as is easily seen) $F^{\prime \prime \prime}$ has nonreal zeros, the nonreal zeros of $F^{(n)}$ for such $F$ (whose existence for large $n$ follows from Pólya's result) already make their appearance no later than $n=3$.

Real meromorphic functions $F$ of the form (0.8) are such that $F$ has only real zeros and real poles, $F^{\prime}$ has no zeros and $F^{\prime \prime}$ has only real zeros. Our main result says that these are the only such transcendental $F$.

THEOREM 1. Let $F$ be a real meromorphic function with only real zeros and real poles (and at least one of each). If $F^{\prime}$ has no zeros and $F^{\prime \prime}$ has only real zeros, then $F$ has one of the forms

$$
F(z)=A \tan (a z+b)+B
$$




$$
\begin{aligned}
& F(z)=(a z+b) /(c z+d), \\
& F(z)=A\left((a z+b)^{2}-1\right) /(a z+b)^{2},
\end{aligned}
$$

where $A, B, a, b, c$ and $d$ are real constants, $a c A \neq 0$, and where, in $(0.11), a d-b c \neq$ 0 .

$\S \S 2,3$ and 4 are devoted to the proof of Theorem 1.

For meromorphic functions $F$ of finite order with real zeros and poles, but no multiple poles, we do not require any hypothesis on the zeros of $F^{\prime \prime}$. In this case we have

THEOREM 2. Let $F$ be meromorphic of finite order, not entire, with only real zeros and poles. Suppose, in addition, that all the poles of $F$ are simple and that $F^{\prime}$ has no zeros. Then, if $F$ is real, it is of the form (0.10) or (0.11). If $F$ is strictly nonreal, it is of the form (0.6).

We shall prove Theorem 2 in $\S 5$.

Applications to differential equations. We will be concerited with the question of the existence of solutions which have only real zeros for the equation

$$
w^{\prime \prime}+H(z) w=0, \quad H \text { entire. }^{2}
$$

If $H$ is constant then (0.13) does have linearly independent solutions which are free of nonreal zeros. However, as we shall show in Theorem 3, if $H$ is a nonconstant polynomial, then such a pair of solutions cannot exist. As a consequence, we may conclude that if $p$ and $q$ are polynomials, then the equation $y^{\prime \prime}+p(z) y^{\prime}+q(z) y=0$ cannot have two linearly independent solutions with only real zeros (provided $2 p^{\prime}+$ $p^{2}-4 q$ is nonconstant) - since the transformation $y=w \exp \left(-\frac{1}{2} \int p\right)$ transforms this latter equation into an equation of the form (0.13).

As we shall see below, there are many instances where $H$ is transcendental entire and $(0.13)$ has two linearly independent solutions with only real zeros. If we require, in addition, that the derivative of one of these solutions has only real zeros, then (Theorem 4) $H$ must have a special form.

On the other hand, by virtue of Theorem A, if $H$ itself has only real zeros then (Theorem 6) (0.13) cannot have any solution which along with its first derivative has only real zeros, unless $H$ is one of four forms. Two of these are classical and may be normalized to $H(z)=z$ for which (0.13) is Airy's equation, and $H(z)=n+1 / 2-z^{2} / 4$ ( $n$ a nonnegative integer) in which case $(0.13)$ is the HermiteWeber equation. The other two forms of $H$ are both transcendental and strictly nonreal. We shall amplify these remarks following the statement of Theorem 6 and in our discussion of Theorem 7.

THEOREM 3. Suppose that $H$ is a polynomial and that $w_{1}$ and $w_{2}$ are linearly independent solutions of (0.13) which have only real zeros. Then $H$ is constant.

The condition that $H$ be a polynomial is necessary. To see this choose an arbitrary nonconstant entire function $h$ and let $H=h^{\prime \prime}-h^{\prime 2}-\exp (4 h)$. Then $w_{1}=\exp \left[-h+\int \exp (2 h)\right]$ and $w_{2}=\exp \left[-h-\int \exp (2 h)\right]$ are solutions of $(0.13)$.

\footnotetext{
${ }^{2}$ The convergence exponent of zeros of solutions of $(0.13)$ has recently been studied by S. Bank and I. Laine, On the oscillation theory of $f^{\prime \prime}+A f=0$ where $A$ is entire, Trans. Amer. Math. Soc. 273 (1982), 357-363.
} 
For transcendental $H$ we have

THEOREM 4. Suppose that $H$ is a transcendental entire function and that $w_{1}$ and $w_{2}$ are linearly independent solutions of $(0.13)$. If $w_{1}, w_{1}^{\prime}$ and $w_{2}$ have only real zeros, then

$$
\begin{gathered}
H(z)=\frac{1}{2} c^{2} e^{i(c z+d)} \cos (c z+d), \\
w_{1}(z)=A \exp \left\{-\frac{1}{2}\left[i(c z+d)+e^{i(c z+d)}\right]\right\}
\end{gathered}
$$

and

$$
w_{2}(z)=A \exp \left\{-\frac{1}{2}\left[i(c z+d)-e^{i(c z+d)}\right]\right\}
$$

$A \neq 0, c \neq 0$ and $d$ constants, with $c$ and $d$ real.

As a consequence of Theorems 3 and 4 we have the

COROLlaRY. Suppose that $H$ is a real nonconstant entire function. If $w_{1}$ and $w_{2}$ are linearly independent solutions of (0.13) and both $w_{1}$ and $w_{1}^{\prime}$ have only real zeros, then $w_{2}$ has nonreal zeros.

If $H$ is real, nonconstant and entire then (0.13) may have a strictly nonreal solution with only real zeros only if $H$ has a particular form. This is the content of

THEOREM 5. Let $H$ be a real nonconstant entire function. Suppose that $(0.13)$ has a strictly nonreal solution $w$ with only real zeros. Then

$$
H(z)=c^{2} e^{-4 s(z)}-s^{\prime \prime}(z)-\left(s^{\prime}(z)\right)^{2}
$$

and

$$
w(z)=\exp \left[s(z)+c i \int e^{-2 s(z)} d z\right],
$$

where $s$ is real entire and $c \neq 0$ is a real constant.

It is readily verified that every function of the form $(0.18)$ is a solution of $(0.13)$ with $H$ given by $(0.17)$.

Returning to the statement of Theorem 4 we note from $(0.14)$ and $(0.15)$ that $H$ as well as $w_{1}$ and $w_{1}^{\prime}$ have only real zeros. Since $w_{1}$ is a solution of $(0.13)$ with $H$ given by $(0.14), w_{1}^{\prime \prime}$ also has only real zeros. Since $w_{1}$ is of the form $(0.15), w_{1}$ is in the class given by $(0.5)$, so that this result is consistent with Theorem A. Indeed, if we assume in (0.13) that $H$ is a nonconstant entire function with only real zeros and that $w$ is a solution which along with $w^{\prime}$ has only real zeros, then $w$ is of the form $(0.1),(0.4)$ or $(0.5)$. Clearly, not every function of the form (0.1) can satisfy (0.13) with $H$ entire. In fact, we have

THEOREM 6. Suppose that $H$ is entire, nonconstant, and has only real zeros. If $(0.13)$ has a solution $w$ such that both $w$ and $w^{\prime}$ have only real zeros, then $H$ takes one of the following four forms:

$$
\begin{aligned}
& H(z)=8 c^{2} e^{3 i(c z+d)} \cos (c z+d), \\
& H(z)=K c^{2} e^{2 i(c z+d)}\left[1-K \sin ^{2}(c z+d)\right], \\
& H(z)=\left(c^{2} / 4\right)\left[2(2 n+1)-(c z+d)^{2}\right],
\end{aligned}
$$




$$
H(z)=c z+d,
$$

where $c \neq 0$ and $d$ are real constants, $K \geq 1$ is constant, and $n$ is a nonnegative integer.

If $H$ is of the form (0.19) then $w$ is given by (0.4) with $2 c$ and $2 d$ in place of $c$ and $d$. In the case of $(0.20)$ we have $w$ of the form (0.5), with $2 c$ and $2 d$ in place of $c$ and $d$, and with $K$ replaced by $-\frac{1}{4} K$. When $H$ is of the form (0.21), equation $(0.13)$ is that of Hermite and Weber, and $w=A H_{n}(c z+d) \exp \left[\frac{1}{4}(c z+d)^{2}\right]$, where $H_{n}$ is the Hermite polynomial of degree $n$. With $H$ as in (0.22) equation (0.13) is Airy's equation and $w$ is a constant multiple of the Airy function $\operatorname{Ai}(c z+d)$. (It is well known that $H_{n}$ has only real zeros. For the reality of the zeros of $\mathrm{Ai}$ we refer the reader to $[10$, pp. 413-415].)

A conjecture of A. Wiman (circa 1915) asserts that if $w$ is real entire with only real zeros and both $w$ and $w^{\prime \prime}$ have only real zeros, then $w$ is in the Laguerre-Pólya class (0.1)-with no hypothesis on $w^{\prime}$. Wiman's conjecture remains unresolved even for functions of the form $w(z)=\exp (Q(z)), Q$ a real polynomial. Equivalently, it remains to be proved that if $P$ is a real polynomial and $P^{\prime}+P^{2}$ has only real zeros, then $P(z)=-a z+b, a \geq 0$.

Our belief in the truth of Wiman's conjecture is our basis for the following

CONJECTURE 2. If $H$ is real entire, nonconstant, with only real zeros and not of the form (0.21) or (0.22), then every solution of (0.13) has nonreal zeros.

If the order $\lambda$ of $H$ satisfies $1<\lambda \leq \infty$, or more generally if $T(r, H) \neq O(r \log r)$, where $T(r, H)$ is the Nevanlinna characteristic of $H$, then Conjecture 2 may be affirmed, as we shall see, by applying the following result of Levin and Ostrovskii $[9$, p. 325], which disposed of the "large" infinite order case for the Pólya problem mentioned at the beginning of our introduction.

THEOREM D. Let $f$ be a real entire function with only real zeros. If the zeros of $f^{\prime \prime}$ form an A-set, then

$$
\log T(r, f)=O(r \log r) \quad(r \rightarrow \infty) .
$$

We remind the reader that a sequence $\left\{a_{n}\right\}$ of complex numbers is an $A$-set if

$$
\sum_{a_{n} \neq 0}\left|\operatorname{Im}\left(a_{n}^{-1}\right)\right|<\infty .
$$

More recently, Shen $[\mathbf{1 3}]$ showed how to exploit the methods of Levin and Ostrovskii to remove the condition of reality both on $f$ and on its zeros. He proved

THEOREM E. Suppose that $f$ is entire. If the zeros of $f f^{\prime \prime}$ form an A-set, then (0.23) holds.

Thus, as we shall prove in $\S 6$, an easy consequence of Theorem $\mathrm{E}$ is the following stronger version of Conjecture 2 when the order of $H$ exceeds one.

THEOREM 7. Let $H$ be an entire function whose zeros form an $A$-set. If

$$
\varlimsup_{r \rightarrow \infty} \frac{T(r, H)}{r \log r}=\infty,
$$

then the sequence of zeros of any solution of (0.13) is not an A-set. 
In particular, every solution of (0.13) has infinitely many nonreal zeros.

It remains, therefore, to investigate Conjecture 2 only for polynomial $H$ and transcendental $H$ of order at most one.

We close this section by recalling our earlier remark that Theorem 3 is equally true for solutions of the equation $y^{\prime \prime}+p(z) y^{\prime}+q(z) y=0$, with $p$ and $q$ polynomials provided $2 p^{\prime}+p^{2}-4 q$ is nonconstant. Theorems 4 and 6 , on the other hand, may fail to generalize to the equation $y^{\prime \prime}+H_{1}(z) y^{\prime}+H_{2}(z) y=0, H_{1}$ and $H_{2}$ entire, since the transformation $y=w \exp \left(-\frac{1}{2} H_{1}\right)$ need not preserve the reality of the zeros of derivatives. Use of this transformation does give an analogue of Theorem 5 for the more general second order equation.

1. Preliminary lemmas. Our proofs depend upon several lemmas concerning entire functions with real zeros and properties of holomorphic maps of the upper half-plane into itself.

Suppose that $\left\{a_{k}\right\}$ and $\left\{b_{k}\right\}$ are sequences of real numbers having the "interlacing" property

$$
a_{k}<b_{k}<a_{k+1} \quad(-\infty \leq \alpha \leq k \leq \omega \leq+\infty, k \text { finite })
$$

and are indexed so that

$$
b_{-1}<0<a_{1}
$$

Now set

$$
\psi(z)= \begin{cases}\frac{z-b_{0}}{z-a_{0}} \prod_{k \neq 0} \frac{1-z / b_{k}}{1-z / a_{k}} & \text { if } \omega=+\infty \\ \frac{z-b_{0}}{\left(z-a_{0}\right)\left(a_{\omega}-z\right)} \prod_{k \neq 0, \omega} \frac{1-z / b_{k}}{1-z / a_{k}} & \text { if } \omega<+\infty\end{cases}
$$

Then we have [8, pp. 308-309].

LEMMA 1. If $\psi(z)$ is of the form (1.3) and $L(z)$ has one of the forms

$$
1, z-b_{\alpha^{\prime}}, b_{\omega}-z,\left(z-b_{\alpha^{\prime}}\right)\left(b_{\omega}-z\right) \quad\left(b_{\alpha^{\prime}}<a_{\alpha}, a_{\omega}<b_{\omega}\right),
$$

then

$$
\psi_{1}(z)=\psi(z) L(z)
$$

maps the upper half-plane into itself.

Now suppose $f$ is a constant multiple of a real entire function with at least one zero. Denote by $\left\{a_{k}\right\}$ its distinct zeros and enumerate them as follows:

$$
\cdots<a_{k-1}<a_{k}<a_{k+1}<\cdots \quad(-\infty \leq \alpha \leq k \leq \omega \leq+\infty, k \text { finite }) .
$$

According to Rolle's theorem, $f^{\prime}$ has at least one zero in each interval $\left(a_{k}, a_{k+1}\right)$; choose one and denote it by $b_{k}$, so that (1.1) is true. Reindexing if necessary, we can assume that (1.2) also holds.

It is clear that the logarithmic derivative of $f$ may be written in the form

$$
\left(f^{\prime} / f\right)(z)=\phi(z) \psi(z),^{3}
$$

where $\phi(z)$ is a real entire function and $\psi(z)$ is of the form (1.3). In fact, we have

\footnotetext{
${ }^{3}$ If $f$ has only one zero, set $\psi(z)=\left(a_{0}-z\right)^{-1}$.
} 
LEMMA 2. $f$ is of finite order if and only if $\phi$ is a polynomial. In particular, $f$ is a Laguerre-Pólya function if $\phi$ is linear.

The proof of this lemma is a consequence of the proofs of Lemma 3 of [4] and Lemmas 2 and 8 of [3].

Our next lemma is a technical one whose proof is by standard growth arguments (cf. [3, Lemma 4]).

LEMMA 3. Let $\Pi(z)$ be a canonical product of genus $p$ with only real zeros, let $\varepsilon>0$, and let

$$
D=\left\{z=r e^{i \theta}: r>0 \text { and } \varepsilon \leq|\theta| \leq \pi-\varepsilon\right\}
$$

Then

$$
\left|\left(\Pi^{\prime} / \Pi\right)(z)\right|=o\left(r^{p}\right) \quad(|z|=r \rightarrow \infty, z \in D) .
$$

The following lemmas follow directly from Hadamard's factorization theorem.

LEMMA 4. Let $g(z)$ be an entire function of genus $\sigma \geq 2$ with only real zeros. Let $M=[\sigma / 2], \varepsilon=\pi / 2(\sigma+1), N$ be any positive integer, and let $D$ be as in (1.6). Then there are at least $M$ rays re $e^{i \psi_{1}}, \ldots, r e^{i \psi_{M}}(0<r<\infty)$ in $D$ such that for $k=1, \ldots, M$

$$
\left|g\left(r e^{i \psi_{k}}\right)\right|=o\left(r^{-N}\right) \quad(r \rightarrow \infty) .
$$

LEMMA 5. Let $g$ be a real entire function with only real zeros of genus 0 or 1 . Suppose $g$ has at least $N$ zeros. Then there exists a constant $c>0$, such that

$$
|g(i y)| \geq c|y|^{N} \quad(-\infty<y<\infty) .
$$

Logarithmic differentiation of the Hadamard representation and usual growth estimates may be used to derive our final preliminary lemma.

LEMMA 6. Let $g$ be real entire of genus 0 or 1 with only real zeros. Then

$$
\left|\left(g^{\prime} / g\right)^{\prime}(i y)\right| \rightarrow 0 \text { as }|y| \rightarrow \infty .
$$

PROOF OF THEOREM 1. We present the proof of this theorem in 3 stages in $\S \S 2,3$ and 4 .

In what follows we shall assume that $F$ is a (constant multiple of a) real meromorphic function satisfying the hypotheses of Theorem 1 . We shall also assume familiarity with the definitions and basic properties of Nevanlinna's functionals, $T(r, F)$ and $m(r, F)$, as well as with those of the less familiar Tsuji characteristic $T_{0}(r, F)$ (cf. e.g. [9]). As usual we will denote by $\lambda=\lambda(G)$ the order of a meromorphic function $G, \lambda=\varlimsup_{r \rightarrow \infty}(\log T(r, G) / \log r)$.

\section{The first reduction.}

LEMMA 7. $F^{\prime} / F=1 / g$, where $g$ is an entire function of order at most one with only real zeros (and at least two of them).

ProOF. Since $F^{\prime}$ has no zeros, $F / F^{\prime}$ is real entire; hence we can write

$$
F / F^{\prime}=g \text {. }
$$

Note that the zeros of $g$ correspond to the zeros and poles of $F$ counted simply. Since $F$ has only real zeros and poles and at least one of each, $g$ has only real zeros 
and at least two of them. Further, since the zeros of $F F^{\prime \prime}$ are real, Lemma D of [6] implies that

$$
T_{0}\left(r, F / F^{\prime}\right)=T_{0}(r, g)=O(\log r) .
$$

Finally, since $g$ is an entire function, it is possible to relate $T_{0}(r, g)$ and $T(r, g)[6$, Lemma $\mathrm{C}$ and Proof of Lemma 1] (cf. also [9, p. 332]) so that (2.2) implies

$$
T(r, g)=O(r \log r) .
$$

It follows from $(2.3)$ that $\lambda(g) \leq 1$.

LEMMA $8 . \lambda(F) \leq 2$.

Proof. Let $H=1 / F^{\prime} . H$ is entire with only real zeros since $F^{\prime}$ has no zeros and only real poles. Thus the logarithmic derivative of $H$ has the form (1.5) and so

$$
-\left(F^{\prime \prime} / F^{\prime}\right)(z)=\left(H^{\prime} / H\right)(z)=\phi(z) \psi(z) .
$$

Since $F^{\prime \prime}$ has only real zeros, (2.4) implies that $\phi$ is real entire with only real zeros. Now (2.1) implies $F^{\prime}=F / g$ so

$$
F^{\prime \prime} / F^{\prime}=F^{\prime} / F-g^{\prime} / g=1 / g-g^{\prime} / g \text {. }
$$

Thus (2.4) and (2.5) yield

$$
\phi \psi=g^{\prime} / g-1 / g .
$$

We will now show that (2.6) implies that $\psi$ is a polynomial of degree at most one. that

Applying standard estimates from the Nevanlinna theory it follows from (2.6)

$$
m(r, \phi) \leq m\left(r, g^{\prime} / g\right)+m(r, 1 / g)+m(r, 1 / \psi)+\log 2 .
$$

Since $g$ is entire of finite order, Nevanlinna's classical lemma on the logarithmic derivative implies

$$
m\left(r, g^{\prime} / g\right)=O(\log r) .
$$

Since $\psi$ has the form (1.3), it maps the upper half-plane into itself by Lemma 1 and therefore satisfies the inequalities of Carathéodory [8, p. 18]

$$
c_{1} r^{-1}|\sin \theta|<\left|\psi\left(r e^{i \theta}\right)\right|<c_{2} r|\sin \theta|^{-1} \quad(r>1, \theta \neq 0, \pi)
$$

where $c_{1}$ and $c_{2}$ are positive constants (depending on $\psi$ ). From this it easily follows that

$$
m(r, 1 / \psi)=O(\log r) .
$$

Thus (2.7), (2.8) and (2.10) imply

$$
m(r, \phi) \leq m(r, 1 / g)+O(\log r) \leq T(r, g)+O(\log r)=m(r, g)+O(\log r)
$$

and therefore

$$
\lambda(\phi) \leq \lambda(g) \leq 1
$$

Since $\lambda(g) \leq 1$ and $g$ has only real zeros, the Hadamard factorization of $g$ and Lemma 3 easily imply

$$
\left|\left(g^{\prime} / g\right)(i y)\right|=o(|y|)
$$


Equations (2.6), (2.9), (2.13) and Lemma 5 now imply

$$
|\phi(i y)|=o\left(|y|^{2}\right) .
$$

Since $\phi$ is real with only real zeros, $(2.12),(2.14)$, Lemma 5 and the Hadamard factorization of $\phi$ imply

$$
\phi(z)=e^{a z}(b z+c),
$$

where $a, b$ and $c$ are real constants. We will show that $a=0$.

Suppose on the contrary that $a<0$. Then, using an argument found in $[\mathbf{4}$, Lemma 3], it follows that $H$ has at most a finite number of positive zeros. Since $H=1 / F^{\prime}$, this implies that $F^{\prime}$ and therefore $F$ has at most a finite number of positive poles. Now $F^{\prime} / F=1 / g$ and so the zeros of $g$ are made up of the zeros and poles of $F$ (each counted simply). But the fact that $F^{\prime}$ has no zeros implies that $F$ has only simple zeros and that the zeros and poles of $F$ interlace. Thus, since $F$ has at most a finite number of positive poles, $g$ has at most a finite number of positive zeros. In addition, (2.5) and the fact that $F^{\prime \prime}$ has only real zeros imply that $g^{\prime}$ has only real one-values. Since $\lambda\left(g^{\prime}-1\right)=\lambda(g) \leq 1, g^{\prime}-1$ is therefore a Laguerre-Pólya function (i.e., has the form (0.1)). It is well known (see e.g. [14, p. 266]) that if $h$ is a Laguerre-Pólya function, the zeros of $h^{(k)}$ which are not also zeros of $h^{(k-1)}$ are real and separate those of $h^{(k-1)}, k=1,2, \ldots\left(h^{(0)}=h\right)$. Since $g$ has at most finitely many positive zeros the same is true of $g^{\prime \prime}=\left(g^{\prime}-1\right)^{\prime}$ and therefore of $g^{\prime}-1$. It now follows from a result of Edrei [1, Corollary, p. 277] that $\lambda(g) \leq \frac{1}{2}$. But then (2.11) implies that $\lambda(\phi) \leq \frac{1}{2}$, which clearly contradicts $(2.15)$ and the assumption that $a<0$. Since a similar argument shows that $a>0$ also leads to a contradiction, we conclude that $a=0$. It then follows from (2.4) and (2.15) (with $a=0$ ) that

$$
\left(H^{\prime} / H\right)(z)=(b z+c) \psi(z) .
$$

Lemma 2 then implies that $H$ is a Laguerre-Pólya function; consequently, $\lambda(H) \leq 2$. Since $F^{\prime}=1 / H$, it follows that

$$
\lambda(F)=\lambda\left(F^{\prime}\right)=\lambda(H) \leq 2 .
$$

This completes the proof of Lemma 8 .

3. The second reduction. As mentioned above, the fact that $F^{\prime}$ has no zeros implies that $F$ has only simple zeros and that the zeros and poles of $F$ separate each other. This in turn implies that we can write

$$
F(z)=\psi_{1}(z) / G(z),
$$

where $\psi_{1}(z)$ is a function of the form (1.4) and $G(z)$ is a (constant multiple of a) real entire function whose zeros are the multiple poles of $F$. By Lemma 1, $\psi_{1}$ maps the upper half-plane to itself and so satisfies the inequalities of Carathéodory (2.9). Thus (3.1) implies

$$
m(r, G) \leq m(r, 1 / F)+m\left(r, \psi_{1}\right) \leq T(r, F)+O(\log r) .
$$

It now follows from Lemma 8 and (3.2) that $\lambda(G) \leq 2$. In fact, we have 
LEMMA 9. $G$ is a constant unless $F$ is of the form (0.12).

Proof. On the one hand, by Lemma $7, F^{\prime} / F=1 / g$, where $g$ is a real entire function of order $\leq 1$ with only real zeros and at least two of them. Hence, by Lemma 5,

$$
\left|\left(F^{\prime} / F\right)(i y)\right|=|1 / g(i y)|=O\left(1 /|y|^{2}\right) .
$$

On the other hand (3.1) implies

$$
\left|\left(F^{\prime} / F\right)(i y)\right| \geq\left|\left(G^{\prime} / G\right)(i y)\right|-\left|\left(\psi_{1}^{\prime} / \psi_{1}\right)(i y)\right| .
$$

We will now show that (3.3) and (3.4) are incompatible unless $G$ is a linear polynomial.

Since $\psi_{1}$ maps the upper half-plane to itself, an application of Schwarz's lemma to $T_{1} \psi_{1} T_{2}^{-1}$, where $T_{1}$ and $T_{2}$ are appropriate linear fractional transformations of the upper half-plane onto the unit disk, shows that

$$
\left|\psi_{1}^{\prime}(z) / \operatorname{Im} \psi_{1}(z)\right| \leq 1 /|\operatorname{Im} z|
$$

so that

$$
\left|\left(\psi_{1}^{\prime} / \psi_{1}\right)(z)\right| \leq 1 /|\operatorname{Im} z|
$$

Since $G$ is a (constant multiple of a) real entire function with only real zeros and $\lambda(G) \leq 2$, we can write

$$
\left(G^{\prime} / G\right)(z)=P(z) \psi(z)
$$

where, by Lemma 2, $P$ is a polynomial and $\psi$ is of the form (1.3). If $G$ is not a Laguerre-Pólya function, then $P(z)$ is a polynomial of degree $\geq 2$ by Lemma 2 . Since $\psi$ satisfies the inequalities (2.9), (3.6) then implies

$$
\left|\left(G^{\prime} / G\right)(i y)\right| \neq o(|y|) .
$$

Combining (3.4), (3.5) and (3.7) we obtain

$$
\left|\left(F^{\prime} / F\right)(i y)\right| \neq o(|y|),
$$

which contradicts (3.3). Thus, $G$ is a Laguerre-Pólya function, and so, by $(0.1)$, we can write

$$
\begin{aligned}
\frac{G^{\prime}}{G}(z) & =\frac{m}{z}+\alpha z+\beta+\frac{\Pi^{\prime}}{\Pi}(z) \\
& =\frac{m}{z}+\alpha z+\beta+z^{p} \sum_{k} \frac{m_{k}}{z_{k}^{p}\left(z-z_{k}\right)},
\end{aligned}
$$

where $p=$ genus $\Pi(z)=0$ or 1 and $m_{k}=$ mulitplicity of the zero $z_{k}$. If $\alpha^{2}+\beta^{2}>0$, Lemma 3 and (3.9) imply

$$
\left|\left(G^{\prime} / G\right)(i y)\right| \neq o(1)
$$

which, combined with (3.4) and (3.5), again contradicts (3.3). Thus $\alpha=\beta=0$ and by $(3.9)$,

$$
\left|\frac{G^{\prime}}{G}(i y)\right| \geq\left|\operatorname{Im} \frac{G^{\prime}}{G}(i y)\right| \geq|y| \sum_{\left|z_{k}\right| \leq|y|} \frac{m_{k}}{y^{2}+z_{k}^{2}}+\frac{m}{|y|}
$$


If $G$ has more than one zero, then (3.11) implies

$$
\varliminf_{|y| \rightarrow \infty}\left|y \frac{G^{\prime}}{G}(i y)\right| \geq 2 .
$$

Combining (3.12) with (3.5) and (3.4) we obtain

$$
\left|\left(F^{\prime} / F\right)(i y)\right| \neq o(1 /|y|) .
$$

Since this contradicts (3.3) we conclude that $G$ is a linear polynomial; i.e.,

$$
G(z)=\gamma z+\delta
$$

To see what happens in the case $\gamma \neq 0$, we assume without loss of generality that $\gamma=1$ and $\delta=0$, so that $F$ has a double pole at $z=0$ (recall from (3.1) that the zeros of $G$ are the multiple poles of $F$ ). Thus, by (3.1),

$$
F(z)=A \psi_{1}(z) / z, \quad A \text { a constant }
$$

and

$$
\left(F^{\prime} / F\right)(z)=\left(\psi_{1}^{\prime} / \psi_{1}\right)(z)-1 / z .
$$

Denote the zeros and poles of $F$ by $\left\{a_{k}\right\}$ and $\left\{b_{k}\right\}$ and index them so that

$$
a_{k}<b_{k}<a_{k+1} \text { and } b_{0}=0 .
$$

Equations (1.3), (1.4), (3.16) and (3.17) thus imply

$$
\begin{aligned}
\operatorname{Im} \frac{F^{\prime}}{F}(i y) & =\operatorname{Im} \frac{\psi_{1}^{\prime}}{\psi_{1}}(i y)-\operatorname{Im} \frac{1}{i y} \\
& =-y \sum_{k}\left[\frac{1}{y^{2}+a_{k}^{2}}-\frac{1}{y^{2}+b_{k}^{2}}\right]+\frac{1}{y} \\
& =\left\{\frac{1}{y}-\frac{y}{y^{2}+a_{0}^{2}}\right\}+y\left\{\sum_{1}+\sum_{2}\right\},
\end{aligned}
$$

where

$$
\sum_{1}=\sum_{k \geq 0}\left[\frac{1}{y^{2}+b_{k}^{2}}-\frac{1}{y^{2}+a_{k+1}^{2}}\right]
$$

and

$$
\sum_{2}=\sum_{k \leq-1}\left[\frac{1}{y^{2}+b_{k}^{2}}-\frac{1}{y^{2}+a_{k}^{2}}\right]
$$

Now, (3.17) implies

$$
1 /\left(y^{2}+b_{k}^{2}\right)-1 /\left(y^{2}+a_{k+1}^{2}\right)>0, \quad k=0,1, \ldots,
$$

and

$$
1 /\left(y^{2}+b_{k}^{2}\right)-1 /\left(y^{2}+a_{k}^{2}\right)>0, \quad k=-1,-2, \ldots
$$

Hence (3.18)-(3.22) yield

$$
\operatorname{Im}\left(F^{\prime} / F\right)(i y) \geq 1 / y-1 /\left(y^{2}+a_{0}^{2}\right) \geq a_{0}^{2} / 2 y^{3} \text { for } y \geq y_{0}>0,
$$


and so, on the one hand,

$$
\left|\left(F^{\prime} / F\right)(i y)\right| \neq o\left(1 / y^{3}\right) \quad(y \rightarrow+\infty),
$$

and, on the other hand, (3.3) and Lemma 5 imply

$$
\left|\left(F^{\prime} / F\right)(i y)\right|=1 /|g(i y)|=o\left(1 / y^{3}\right) \quad(y \rightarrow+\infty)
$$

if $g$ has more than three zeros. Since this is incompatible with (3.24), $g$ has two or three zeros. This implies that there are 3 possibilities:

(a) $F$ has one zero and a (double) pole at $z=0$,

(b) $F$ has one zero and two distinct poles (one of which is a double pole at $z=0$ ), or

(c) $F$ has two (distinct) zeros and a (double) pole at $z=0$.

Say (b) occurs, and $b_{0}, b_{1}$ are the poles, $a_{1}$ the zero, and $0=b_{0}<a_{1}<b_{1}$. Then, it follows easily from (3.18), (3.19) and (3.21) that

$$
\operatorname{Im}\left(F^{\prime} / F\right)(i y) \neq o(1 / y) \quad(y \rightarrow+\infty) .
$$

But then (3.25) becomes

$$
\left|\left(F^{\prime} / F\right)(i y)\right|=1 /|g(i y)| \sim C / y^{3}
$$

for some constant $C \neq 0$ since $g$ now has three zeros. Since (3.26) and (3.27) are incompatible, case (b) cannot occur. A similar analysis shows that (a) cannot occur either. Thus (c) remains, and we have either

$$
F(z)=A(z+a)(z-b) / z^{2}, \quad \text { where } a, b>0,
$$

or

$$
F(z)=A \psi_{1}(z), \quad A \text { a constant. }
$$

Note that if $F$ is of the form (3.28), F, $F^{\prime}$ and $F^{\prime \prime}$ have only real zeros. The requirement that $F^{\prime}$ have no zeros forces $a=b$ so that either

$$
F(z)=A\left(z^{2}-a^{2}\right) / z^{2}
$$

or

$$
F(z)=A \psi_{1}(z)
$$

Allowing $z$ to undergo a real affine transformation, we see that (3.30) is equivalent to $(0.12)$. This completes the proof of Lemma 9 .

4. The final reduction. We will now show that if $F$ is of the form (3.31) then, in fact, $F$ must have one of the forms $(0.10)$ or $(0.11)$. To do this, we will use the Schwarzian derivative $\{F, z\}$ defined by

$$
\{F, z\}=\left(F^{\prime \prime} / F^{\prime}\right)^{\prime}-\frac{1}{2}\left(F^{\prime \prime} / F^{\prime}\right)^{2} .
$$

If we use the fact that $F$ is of finite order (Lemma 8) and apply Nevanlinna's classical lemma on the logarithmic derivative together with some standard estimates, then we can deduce from (4.1) that

$$
m(r,\{F, z\})=O(\log r) .
$$


But, since $F$ has only simple zeros and poles and $F^{\prime}$ has no zeros, $\{F, z\}$ is entire. So (4.2) implies that $\{F, z\}$ is a polynomial, say

$$
\{F, z\}=2 P(z) \text {. }
$$

Now we know from Lemma 7 that $F^{\prime} / F=1 / g$, where $g$ is an entire function with only real zeros. This implies

$$
\begin{aligned}
\{F, z\} & =-g^{\prime \prime} / g+\frac{1}{2}\left(g^{\prime} / g\right)^{2}-\frac{1}{2}\left(1 / g^{2}\right) \\
& =-g^{\prime \prime} / g^{\prime} \cdot g^{\prime} / g+\frac{1}{2}\left(g^{\prime} / g\right)^{2}-\frac{1}{2}\left(1 / g^{2}\right) .
\end{aligned}
$$

Since $\lambda(g) \leq 1$, it follows easily from (4.4), Lemma 3 and the Hadamard factorization of $g$ and $g^{\prime}$ that

$$
\{F, z\}(i y)=o\left(|y|^{2}\right) \text {. }
$$

This implies that $P(z)$ is of degree $\leq 1$. Thus (4.3) yields

$$
\{F, z\}=2(a z+b), \quad a, b \text { real constants. }
$$

We will now show that $a=0$.

To do this, we consider the differential equation associated with (4.6),

$$
y^{\prime \prime}(z)+(a z+b) y(z)=0 .
$$

If $a \neq 0$, we can, if necessary, allow $z$ to undergo a real affine transformation and rewrite (4.7) as

$$
w^{\prime \prime}(z)-z w(z)=0,
$$

which is Airy's equation. It is known $[\mathbf{1 5}, \mathrm{pp} .69-70]$ that every nonzero solution of (4.8) is an entire function of order $\frac{3}{2}$. It is also known $[\mathbf{7}$, p. 375] that every nonzero solution of (4.6) is of the form

$$
F=w_{1} / w_{2},
$$

where $w_{1}$ and $w_{2}$ are linearly independent solutions of (4.8). Moreover, the nature of (4.8) dictates the constancy of the Wronskian of $w_{1}$ and $w_{2}$, so that logarithmic differentiation of (4.9) gives

$$
F^{\prime} / F=C / w_{1} w_{2}, \quad C \text { a constant. }
$$

Since $w_{1}$ and $w_{2}$ are of order $\frac{3}{2}$, so is $w_{1} w_{2}$ and in turn also $F^{\prime} / F$, which contradicts Lemma 7. Consequently, we must have $a=0$.

By (4.6) then, the Schwarzian derivative of $F$ can be written as

$$
\{F, z\}=2 b
$$

and, by (4.7) the associated differential equation as

$$
y^{\prime \prime}+b y=0 \text {. }
$$

Since (4.11) is particularly easy to solve, we again use the relationship between $\{F, z\}$ and linearly independent solutions to (4.11) to find, after allowing $z$ to undergo a real affine transformation, that if $b=k^{2} \geq 0$, then $F$ is either of the form $(0.10)$ or $(0.11)$. If $b<0$, we find that all solutions of $(4.10)$ which are not entire have infinitely many nonreal poles.

This completes the proof of Theorem 1 . 
5. Proof of Theorem 2. Without loss of generality we assume that $F(0)=1$.

We first consider the case of $F$ real. Since $F$ is real with only real zeros and poles, all poles simple, and $F^{\prime}$ has no zeros it follows that the zeros of $F$ are also simple and interlace the poles of $F$. Since $F$ is of finite order we may express $F$ by

$$
F(z)=e^{Q(z)} \psi(z),
$$

where $Q$ is a real polynomial and $\psi$ is of the form (1.3). Logarithmic differentiation gives

$$
F^{\prime} / F=Q^{\prime}+\psi^{\prime} / \psi=1 / g,
$$

where $g$ is real entire of finite order with only real zeros.

In view of Lemma 4 and (3.5) with $\psi$ in place of $\psi_{1}$, it follows that (5.2) can hold only if $Q^{\prime}=0$ and $g$ is of genus 0 or 1 . Then it follows that $F=A \psi, A$ a constant. This is precisely the form of $F$ treated in $\S 4$. Since $g$ has genus 0 or 1 , we may repeat the argument of $\S 4$ to show that $F=w_{1} / w_{2}$, where $w_{1}$ and $w_{2}$ are solutions in the Laguerre-Pólya class of equation (4.7). The constancy of the Wronskian of $w_{1}$ and $w_{2}$ leads to $F^{\prime \prime}=c w_{2}^{\prime} / w_{2}^{3}, c$ a constant. Since $w_{2}^{\prime}$ is also a Laguerre-Pólya function, $F^{\prime \prime}$ has only real zeros. Applying Theorem 1 we conclude that $F$ is either of the form $(0.10)$ or $(0.11)$.

We assume now that $F$ is not a constant multiple of a real function. We write

$$
F=e^{P_{1}+i P_{2}} \Pi_{1} / \Pi_{2},
$$

where $P_{1}$ and $P_{2}$ are real polynomials, $P_{2}$ nonconstant, and $\Pi_{1}$ as well as $\Pi_{2}$ are canonical products of finite genus with only real zeros. Since $F^{\prime}$ is zero free,

$$
F^{\prime} / F=i P_{2}^{\prime}+P_{1}^{\prime}+\Pi_{1}^{\prime} / \Pi_{1}-\Pi_{2}^{\prime} / \Pi_{2}=e^{i Q} / g,
$$

where $g$ is real entire of finite order with only real zeros and $Q$ is a real polynomial. Equating imaginary parts in (5.4) on the real axis we deduce thi $t$ for all $z$,

$$
P_{2}^{\prime}(z)=(\sin Q(z)) / g(z) .
$$

From (5.5) we see that all but finitely many of the zeros of $\sin Q(z)$ must be zeros of $g$. Since $g$ has only real zeros, we must have $Q$ linear, say

$$
Q(z)=\alpha z+\beta \quad(\alpha, \beta \text { real }) .
$$

From (5.4)-(5.6) we obtain

$$
\left(F^{\prime} / F\right)(z)=e^{i(\alpha z+\beta)} P_{2}^{\prime}(z) / \sin (\alpha z+\beta) .
$$

Recalling our assumption that $F$ has only simple poles and that $F^{\prime}$ has no zeros, a simple residue consideration shows that either $P_{2}^{\prime}$ is constant or $\alpha=0$. Since $F$ has zeros as well as poles, $\alpha \neq 0$. So $P_{2}^{\prime}=K$, a real constant, and we infer from (5.7) that

$$
\left(F^{\prime} / F\right)(z)=K e^{i(\alpha z+\beta)} / \sin (\alpha z+\beta) .
$$

Integrating, we find that

$$
F(z)=A e^{i K z} \sin ^{K / \alpha}(\alpha z+\beta) .
$$

In order that $F$ be meromorphic with at least one pole, and all poles simple, we must have $K=-\alpha$. This completes our proof. 


\section{Proofs of Theorems 3-7.}

Theorem 3. We note first from the form of $(0.13)$ that the Wronskian of $w_{1}$ and $w_{2}$ is constant. We suppose now that $w_{2}$ also has only real zeros. Set

$$
F=w_{2} / w_{1} \text {. }
$$

Since $w_{1}$ is a nonzero solution of $(0.13)$ its zeros (if any) are simple, so that $F$ is meromorphic with real zeros and real simple poles. Differentiating (6.1) and using the constancy of the Wronskian gives

$$
F^{\prime}=C / w_{1}^{2}, \quad C \text { a constant, }
$$

along with the fact that $F^{\prime}$ has no zeros. Since $H$ is a nonconstant polynomial, $w_{1}$ is entire of finite order at least $\frac{3}{2}$. The same is true therefore for the order of $F^{\prime}$, and thus for $F$. Because an entire function of finite order greater than one and its derivative cannot both be zero free, it follows from (6.1) and (6.2) that either $w_{1}$ or $w_{2}$ has a zero. We assume that $w_{1}$ has a zero. (Otherwise, we interchange $w_{1}$ and $w_{2}$ in (6.1) and (6.2).) Then $F$ satisfies the hypotheses of Theorem 2, and we have a contradiction, since the order of $F$ exceeds 1 .

Theorem 4. Suppose that $w_{1}, w_{1}^{\prime}$ and $w_{2}$ all have only real zeros. As in the proof of Theorem 3 we again define $F$ by (6.1) and observe that (6.2) holds. Differentiating (6.2) gives

$$
F^{\prime \prime}=-2 C w_{1}^{\prime} / w_{1}^{3} .
$$

From (6.1), (6.2), (6.3) and our assumptions on $w_{1}, w_{1}^{\prime}$ and $w_{2}$, we deduce that $F$ is meromorphic with real zeros and real simple poles, that $F^{\prime}$ has no zeros, and that $F^{\prime \prime}$ has only real zeros. Having assumed that $H$ is transcendental and that $w_{1}$ is a solution of $(0.13)$, standard growth properties imply that $w_{1}$ has infinite order and, because of $(6.2)$, that $F$ is of infinite order. Suppose first that $F$ is a constant multiple of a real function. If $w_{1}$ and $w_{2}$ each have a zero then $F$ satisfies the hypotheses of Theorem 1 and since the order of $F$ is infinite we have a contradiction. If $w_{1}$ has no zeros then $F$ is real entire (up to a constant factor) and $F, F^{\prime}$ and $F^{\prime \prime}$ have no nonreal zeros. For $F$ of infinite order this is impossible by Theorem A. Assume now that $w_{1}$ has a zero but $w_{2}$ has no zeros. Again $F, F^{\prime}$ and $F^{\prime \prime}$ have only real zeros. Since $F$ is a constant multiple of the reciprocal of a real transcendental entire function, this too is impossible, by virtue of Theorem C.

We are left with the possibility that $F$ is a strictly nonreal meromorphic function of infinite order which has only real zeros and real (simple) poles, if any, with $F^{\prime}$ having no zeros and $F^{\prime \prime}$ only real zeros. From Theorems A and B we see that $F$ can only be of the form

$$
F(z)=K \exp \left(e^{i(c z+d)}\right)
$$

$K \neq 0, c \neq 0$ and $d$ constants, with $c$ and $d$ real. From (6.2) and (6.4) we derive

$$
w_{1}=A \exp \left[-\frac{i}{2}(c z+d)-\frac{1}{2} e^{-i(c z+d)}\right], \quad A \neq 0 \text { constant. }
$$

We now combine $(6.1),(6.4)$ and $(6.5)$ to find $w_{2}$. To determine the form of $H$ we substitute $w_{1}$ from (6.5) into (0.13).

Theorem 5 . Let $w$ be a strictly nonreal solution of $(0.13)$ with only real zeros. Then

$$
w=e^{i g} h
$$


where $g$ and $h$ are real entire with $g$ nonconstant and $h \neq 0$. Since $H$ is real entire,

$$
\bar{w}=e^{-i g} h
$$

is also a solution of (0.13). Using the constancy of the Wronskian of $w$ and $\bar{w}$ we find that

$$
g^{\prime} h^{2}=c, \quad c \text { a real constant. }
$$

Having assumed that $g$ is nonconstant we have $g^{\prime} \neq 0$. In addition $h$ must be zero-free; otherwise by (6.8) we would have $h=0$, which is impossible since $w \neq 0$. Thus

$$
h=e^{s}, \quad s \text { real entire, }
$$

and in view of $(6.8)$

$$
g=c \int e^{-2 s}
$$

From (6.6), (6.9) and (6.10) we obtain (0.18). Then (0.17) follows from (0.18) and (0.13).

Theorem 6. As we remarked in the introduction, our hypothesis implies that $w$ is entire of order at least $\frac{3}{2}$ and $w, w^{\prime}$, as well as $w^{\prime \prime}$ have only real zeros. Invoking Theorem A, we must have $w$ of the form $(0.1),(0.4)$ or $(0.5)$. It is easily verified that a solution to (0.13) of the form (0.4) corresponds to $H$ of the form (0.19), and that a solution of the form (0.5) leads to $H$ of the form $(0.20)$. It remains to determine the nonconstant entire $H$ for which $(0.13)$ has a solution in the Laguerre-Pólya class, i.e. of the form (0.1).

We recall that growth properties of solutions of $(0.13)$ dictate $[15$, pp. 69-70] that we may have a solution of Laguerre-Pólya type only if $H$ is a linear or quadratic polynomial. In the former case we obtain $(0.22)$. We consider the quadratic case.

Let

$$
H(z)=\alpha z^{2}+\beta z+\gamma, \quad \alpha \neq 0, \beta, \gamma \text { real constants, }
$$

in (0.13). Assume that $w$ is a solution of the form (0.1). Then

$$
-H=w^{\prime \prime} / w=\left(w^{\prime} / w\right)^{\prime}+\left(w^{\prime} / w\right)^{2} .
$$

Put

$$
w=e^{-a z^{2}} g(z),
$$

where $a \geq 0$ and $g$ is real entire of genus 0 or 1 with only real zeros. From (6.11), (6.12), (6.13) and Lemmas 3 and 6, we deduce upon setting $z=i y$ that

$$
-\alpha y^{2}+\beta i y+\gamma=4 a^{2} y^{2}+o\left(y^{2}\right) \quad \text { as }|y| \rightarrow \infty
$$

so that

$$
\alpha=-4 a^{2} \text { and } a>0 ;
$$

since $\alpha \neq 0$ and $a \geq 0$. We observe next that elementary considerations imply that if $H$ is given by (6.11) with $\alpha<0$ then every nonconstant solution of $(0.13)$ has only 
finitely many real zeros. Since $w$ has only real zeros, $g$ in (6.13) is a polynomial, say $P$. We rewrite $(6.13)$ as

$$
w(z)=P(z) e^{-a z^{2}+b z}, \quad a>0, b \text { real, } P \neq 0 .
$$

Without loss of generality, a real affine transformation permits us to consider, instead of (6.16), a solution to (0.13) of the form

$$
w(z)=P(z) e^{-z^{2} / 4}, \quad P \neq 0 \text { a polynomial, }
$$

and, with possibly other values of $\beta$ and $\gamma,(6.11)$ becomes

$$
H(z)=-\frac{1}{4} z^{2}+\beta z+\gamma, \quad \beta \text { and } \gamma \text { real. }
$$

Substituting from (6.17) and (6.18) into (0.13) gives

$$
\beta z P-z P^{\prime}+(\gamma-1 / 2) P+P^{\prime \prime}=0 .
$$

Set

$$
P(z)=\sum_{k=0}^{n} c_{k} z^{k}, \quad n \geq 0 \text { and } c_{n} \neq 0 .
$$

Replace $P$ in (6.19) by (6.20) and equate the coefficients of $z^{n+1}$ and $z^{n}$ to 0 . We obtain, since $c_{n} \neq 0$,

$$
\beta=0 \text { and } \gamma=n+\frac{1}{2} .
$$

From (6.11), (6.15) and (6.21) we arrive at

$$
H(z)=-\frac{1}{4} z^{2}+n+\frac{1}{2}, \quad n \geq 0 \text { an integer. }
$$

It is well known that for (6.17) to be a solution of $(0.13)$ with $H$ given by $(6.22)$, we must have

$$
P(z)=A H_{n}(z)
$$

where $H_{n}$ is the Hermite polynomial of degree $n$.

With $H$ in (0.13) given by (6.22) our conclusion follows upon using the transformation $z \rightarrow c z+d$.

Theorem 7. We show first that if $w$ is a nonzero solution of $(0.13)$ with $H$ satisfying (0.25), then

$$
\varlimsup_{r \rightarrow \infty} \frac{\log T(r, w)}{r \log r}=\infty .
$$

Suppose (6.24) is false. Then there exists $K<\infty$ such that

$$
\log T(r, w) \leq K r \log r \quad(r \geq 2) .
$$

Since $w$ is a solution of $(0.13)$, we have

$$
-H=\frac{w^{\prime \prime}}{w}=\frac{w^{\prime \prime}}{w^{\prime}} \frac{w^{\prime}}{w} .
$$

Since $H$ is entire, the relation (6.26) together with standard inequalities of Nevanlinna's theory, including particularly his important inequality for the logarithmic 
derivative [2, p. 36], show that for suitable constants $C$ and $C^{\prime}$ and $r>r_{0}$,

$$
\begin{aligned}
T(r, H) & =m(r, H) \leq m\left(r, w^{\prime \prime} / w^{\prime}\right)+m\left(r, w^{\prime} / w\right) \\
& \leq C\left[\log m\left(2 r, w^{\prime}\right)+\log m(2 r, w)+\log r\right] \\
& \leq C\left[\log m\left(2 r, w^{\prime} / w\right)+2 \log m(2 r, w)+\log r\right] \\
& \leq C^{\prime}[\log m(4 r, w)+\log r] .
\end{aligned}
$$

Noting that $m(4 r, w)=T(4 r, w)$ and combining (6.27) with (6.25) we obtain a contradiction of (0.25). Thus (6.24) must hold.

To complete the proof of Theorem 7 we observe next that if the zeros of both $H$ and $w$ form an $A$-set then so do the zeros of $H w$. It follows that if the zeros of $H$ form an $A$-set and $w$ is a solution of (0.13) whose zeros form an $A$-set, then the same is true for the zeros of $w^{\prime \prime}$. From Theorem $\mathrm{E}$ we know that this cannot occur if (6.24) holds. Since (6.24) is a consequence of (0.13) and (0.25) our theorem is proved.

\section{REFERENCES}

1. A. Edrei, Meromorphic functions with three radially distributed values, Trans. Amer. Math. Soc. 78 (1955), 276-293.

2. W. K. Hayman, Meromorphic functions, Oxford Math. Mono., Clarendon Press, Oxford, 1964.

3. S. Hellerstein and J. Williamson, Derivatives of entire functions and a question of Pólya, Trans. Amer. Math. Soc. 227 (1977), 227-249.

4. __ Derivatives of entire functions and a question of Pólya. II, Trans. Amer. Math. Soc. 234 (1977), 497-503.

5. Soc. 263 (1981), 501-513.

6. S. Hellerstein, L. C. Shen and J. Williamson, Reality of the zeros of an entire function and its derivatives, Trans. Amer. Math. Soc. 275 (1983), 319-331.

7. E. Hille, Ordinary differential equations in the complex domain, Wiley, New York, 1976.

8. B. Ja. Levin, Distribution of zeros of entire functions, Transl. Math. Mono., vol. 5, Amer. Math. Soc, Providence, R.I., 1964.

9. B. Ja. Levin and I. V. Ostrovskii, The dependence of the growth of an entire function on the distribution of the zeros of its derivatives, Amer. Math. Soc. Transl. (2) 32 (1963), 322-357.

10. F. W. J. Olver, Asymptotics and special functions, Academic Press, New York, 1974.

11. G. Pólya, On the zeros of the derivatives of a function and its analytic character, Bull. Amer Math. Soc. 49 (1943), 178-191.

12. J. Rossi, The reciprocal of an entire function of infinite order and the distribution of the zeros of its second derivative, Trans. Amer. Math. Soc. 270 (1982), 667-683.

13. L. C. Shen, On the dependence of the growth of an entire function on the distribution of the zeros of its derivatives and a conjecture of Pólya, Ph.D. Dissertation, University of Wisconsin, 1981.

14. E. C. Titchmarsh, The theory of functions, 2nd ed., Oxford Univ. Press, London, 1939.

15. H. Wittich, Neuere Untersuchungen über eindeutige analytische Funktionen, 2nd ed., SpringerVerlag, Berlin and New York, 1968.

Department of MATHEMATICS, University of WisConsin, MADison, Wisconsin 53706 (Current address of Simon Hellerstein)

Department of Mathematics, California institute of Technology, PasaDENA, CALIFORNIA 91225

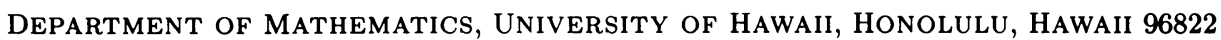
(Current address of Jack Williamson)

Current address (Li-Chien Shen): Department of Mathematics, Syracuse University, Syracuse, New York 13210 